\title{
EL CUESTIONAMIENTO COMO HERRAMIENTA FUNDAMENTAL PARA EL DESARROLLO DE LA TOMA DE DECISIONES DE LOS ALUMNOS EN EDUCACIÓN FÍSICA
}

\author{
QUESTIONING AS A FUNDAMENTAL TOOL FOR PHYSICAL EDUCATION \\ STUDENTS TO DEVELOP DECISION-MAKING SKILLS
}

O QUESTIONAMENTO COMO FERRAMENTA FUNDAMENTAL PARA O

DESENVOLVIMENTO DA TOMADA DE DECISÃO DE ESTUDANTES EM

EDUCAÇÃO FÍSICA.

\author{
Vicente María Gaspar Gil*, Fernando Del Villar Álvarez**, \\ Alba Práxedes Pizarro***, Alberto Moreno Domínguez*
}

\section{Palabras clave:}

TGfU. Educación Primaria. Baloncesto.
Toma de decisiones.

Resumen: El objetivo del presente estudio fue analizar el efecto de un programa de enseñanza comprensiva, basado en el cuestionamiento, sobre la toma de decisiones en las habilidades del pase y del lanzamiento, en una unidad didáctica de baloncesto en Educación Primaria. Participaron 37 alumnos de 6ํㅡㄹ de Primaria con edades comprendidas entre los 11 y 12 años. La variable independiente fue el programa de intervención, basado en el modelo Teaching Games for Understanding (TGfU). La variable de toma de decisiones fue evaluada a través de la observación sistemática. Los resultados obtenidos mostraron que, tras la intervención, los alumnos que recibieron el cuestionamiento durante el desarrollo de las actividades formativas mejoraron su toma de decisiones en comparación con los alumnos que no lo recibieron. Estos resultados manifiestan la necesidad de incluir este modelo pedagógico en las programaciones docentes en los niveles superiores de la etapa de Primaria.

Palavras chave TGfU.

Tomada de decisão. Ensino fundamental. Basquetebol.

Keywords:

TGfU.

Decision-making Education, Primary. Basketball.
Resumo: O principal objetivo do estudo foi analisar o efeito de um programa de ensino centrado na compreensão, baseado no questionamento, na tomada de decisões nas ações de passe e do lançamento, numa unidade didática de basquetebol no ensino fundamental. Participaram 37 alunos com idades compreendidas entre 11 e 12 anos. A variável independente foi o programa de intervenção baseado no modelo Teaching Games for Understanding (TGfU). A variável dependente de tomada de decisão foi avaliada através da observação sistemática. Os resultados mostraram que, após a intervenção, os alunos que receberam o questionamento durante o desenvolvimento das atividades de treino melhoraram sua tomada de decisão em relação aos alunos que não 0 receberam. Esses resultados mostram a necessidade de incluir esse modelo pedagógico nos programas de ensino nos níveis superiores do ensino fundamental.

\footnotetext{
Abstract: The main purpose of this study was to analyze the effect of a comprehensive questioning-based teaching program about decision-making on passing and throwing abilities in a Primary Education teaching unit focused on basketball. Thirty-seven students aged 11-12 participated in the study. The independent variable was the intervention program based on the Teaching Games for Understanding (TGfU). The decision-making variable was assessed by systematic observation. The results obtained showed that, after the intervention, the students who received the questioning when developing training activities improved their decision-making compared to those who did not. These results show the need to include this pedagogical model in teaching programs at the higher levels of Primary Education.
}

*Universidad de Extremadura. Cáceres, España.

E-mail: vicentegaspargil@gmail.com; amorenod@unex.es

**Universidad Rey Juan Carlos. Madrid, España.

E-mail: fernando.delvillar@urjc.es

***Universidad de Nebrija. Madrid, España.

E-mail: apraxedes@nebrija.es

Recebido em: 10-09-2018

Aprovado em: 22-03-2019

Publicado em: 14-05-2019

DOI

https://doi.org/10.22456/1982-8918.86547

(c) (1) (8) Licence 


\section{INTRODUCCIÓN}

Durante mucho tiempo, la enseñanza del deporte en el área de Educación Física (EF) se ha llevado a cabo bajo un enfoque tradicional, que ha descontextualizado la enseñanza de las habilidades deportivas abiertas ${ }^{1}$, dado que la ejecución técnica es practicada de forma aislada a la situación real del juego. Esta situación genera un deficiente desarrollo de las habilidades cognitivas, tales como la toma de decisiones (ALLISON; THORPE, 1997; GRAY; SPROULE, 2011), y de una escasa comprensión del propósito o aplicación de la acción (SALTER, 1999). Hopper (2002), manifiesta que las técnicas aplicadas en aislamiento no se transfieren a situaciones reales del deporte, ya que se basan en la mera repetición, siendo éste un verdadero problema en los modelos tradicionales, ya que desarrollan muy poco la capacidad para comprender la propia naturaleza de los juegos. Este hecho acontece igualmente en etapas educativas tempranas (Educación Primaria ${ }^{2}$ ), en el que se introducen habilidades deportivas técnico-tácticas sin atender a un primer desarrollo de la comprensión del juego.

En este sentido, cabe destacar que el desarrollo de las variables cognitivas son particularmente relevantes, ya que determinan el logro de las habilidades motrices que deben pretender alcanzar los alumnos en las clases de EF (VICKERS; REEVES; CHAMBERS; MARTELL, 2004). Su tratamiento desde el área de EF debe ser esencial, ya que las habilidades cognitivas de los alumnos mejorarán a medida que aumente el número de experiencias tácticas (GARCÍA-GONZÁLEZ; MORENO; MORENO; GIL; DEL VILLAR, 2013).

Así, la manera de huir de los requerimientos puramente técnicos, para valorar la importancia que tiene atender y valorar los procesos cognitivos y decisionales en la enseñanza de deportes colectivos, es incorporar los modelos alternativos a la escuela, debido a la gran similitud que estos tienen con las teorías constructivistas (BUTLER; OSLIN; MITCHELL; GRIFFIN, 2008). En esta línea, estos modelos se presentan como una alternativa eficaz para el desarrollo de la toma de decisiones de los alumnos (BALAKRISHNAN; RENGASAMY; AMAN, 2011; TALLIR; LENOIR; VALCKE; MUSCH, 2007), tales como, Tactical Games Models (GRIFFIN; MITCHELL; OSLIN, 1997), Play Practice (LAUNDER, 2001), Concept Based Games (MCNEILL; FRY; WRIGHT; TAN; TAN; SCHEMPP, 2004), Teaching Games for Understanding (TGFU) (BUNKER; THORPE, 1982), entre otros.

De este modo, en los últimos años, el proceso de enseñanza-aprendizaje de los deportes se ha visto favorecido por la inclusión de modelos pedagógicos centrados en la comprensión del juego, que estimulan a los alumnos a resolver problemas mediante la utilización de su experiencia, a medida que se garantiza una mayor implicación en las actividades deportivas desarrolladas (GUTIÉRREZ; GARCÍA-LÓPEZ, 2012; SINGLETON, 2009). Algunas de las ideas iniciales de estos nuevos modelos pedagógicos fueron el uso de pequeños juegos, la organización de la enseñanza a partir de los principios del juego y la adaptación del material para facilitar la ejecución del alumnado con menor habilidad (ÚBEDA-COLMENAR; MONFORTE; DAVID-DAVID, 2017). Esta ejecución en las situaciones de juego responde a una solución mental previa que viene definida por la capacidad de percepción y de análisis de los deportistas, denotándose fundamental el desarrollo de la acción táctica con el fin de favorecer una enseñanza centrada en la comprensión del juego (MAHLO, 1970).

1 Habilidades deportivas que se desarrollan en condiciones ambientales cambiantes, es decir, en las que las condiciones del entorno varían durante la ejecución (POULTON, 1957). Son las habilidades deportivas de modalidades en las que existe oposición y/o colaboración (como el pase en baloncesto, por ejemplo), cuyos elementos del entorno de juego condicionan el modo en el que se desarrollan.

2 Ensino Fundamental II 
En este sentido, Bunker y Thorpe (1982) desarrollaron este enfoque de enseñanza contextual centrado en entender el juego, mediante el modelo TGfU, reduciendo las demandas técnicas y modificando las condiciones de la práctica, para que los alumnos sean capaces de desarrollar un entendimiento de los aspectos tácticos y, a partir de ahí, poder seguir avanzando en el aprendizaje del juego, mejorando los aspectos tanto técnicos y tácticos.

El modelo TGfU no solo se sitúa como uno de los modelos pedagógicos más favorables para mejorar la capacidad de tomar decisiones en un contexto deportivo (GRIFFIN; BROOKER; PATTON, 2005; PRÁXEDES; MORENO; SEVIL; GARCÍA-GONZÁLEZ; DEL VILLAR, 2017), sino que también ha sido avalado por numerosos autores como un modelo eficaz en contextos educativos (BALAKRISHNAN et al., 2011; HARVEY; GITTINS, 2014; PRÁXEDES; GARCÍAGONZÁLEZ; MORENO-CORTÉS; MORENO; MORENO, 2016; LÓPEZ; PRÁXEDES; DEL VILLAR, 2016; ÚBEDA-COLMENAR; MONFORTE; DAVID-DAVID, 2017).

El modelo TGfU se basa en los principios pedagógicos del juego modificado (juego modificado por representación y juego modificado por exageración) y en el cuestionamiento (THORPE; BUNKER; ALMOND, 1986). Con respecto al primer principio pedagógico, los juegos modificados permiten exponer al alumno a situaciones similares al contexto real, adaptados al nivel de desarrollo de los aprendices, pudiendo realizar modificaciones en cuanto a las reglas, número de jugadores, áreas de juego y equipamiento (HARVEY; CUSHION; MASSAGONZÁLEZ, 2010). En una situación real de juego, se permite que el alumno aprecie el concepto de juego, desarrolle su conciencia táctica, compruebe la relevancia de las habilidades en las distintas situaciones de juego, obtenga una comprensión de cómo jugar y actuar en un deporte y permitimos que los alumnos seleccionen la mejor decisión (KIRK; BROOKER; BRAIUKA, 2000).

El segundo principio pedagógico del modelo TGfU es el cuestionamiento, herramienta que ha resultado ser muy útil, ya que provoca mejoras, tanto en la ejecución motriz de una habilidad técnica, como en las variables decisionales y tácticas en deportes de carácter abierto (GIL; DEL VILLAR, 2014).

El cuestionamiento es una técnica orientada principalmente al alumno y en su capacidad de resolver los problemas que van apareciendo durante el juego (WEBB; PEARSON; FORREST, 2006). En este contexto, el profesorado sirve de guía que ayuda a resolver los problemas tácticos del juego con la idea de reducir dicha ayuda progresivamente para que el alumnado gane autonomía y responsabilidad (SINGLETON, 2009; LIGHT; FAWNS, 2003). El profesorado debe hacer preguntas durante el juego, pero dejando jugar al alumno, para recurrir posteriormente a pequeños debates que estimulen el pensamiento táctico, de tal forma que ayude al alumnado a analizar el juego y a buscar soluciones en la práctica (BUNKER; THORPE, 1982; PEARSON; WEBB, 2008). Los docentes deben favorecer el intercambio de preguntas y respuestas, ya que éste facilita el uso de discusiones sobre los juegos deportivos (HA; WANG; COLLINS, 2014).

En este sentido, Griffin (1996) señala que tanto las discusiones de grupo como la toma de decisiones, inducidas ambas por las preguntas del docente, contribuyen de manera decisiva al desarrollo personal del alumnado. Por ello, los períodos de preguntas y respuestas durante el juego no debe ser una actividad separada del proceso de enseñanza-aprendizaje ya que ayudan a los alumnos a reflexionar sobre lo que se ha hecho y por qué se ha hecho (WRIGHT; MCNEILLL; BUTLER, 2004). Con la utilización del cuestionamiento, se pretende así que los 
alumnos sean conscientes de los puntos importantes que deben recordar para poder realizar satisfactoriamente una tarea, aplicándolos, siempre que sea necesario y pertinente, en tareas de mayor o menor dificultad. En este sentido, hay que destacar que el cuestionamiento es una herramienta que ha dado lugar a mejoras en la toma de decisiones de los alumnos (GARCÍAGONZÁLEZ et al., 2013; PRÁXEDES et al., 2016), permitiendo un mayor desarrollo de la experiencia cognitiva de los estudiantes (GIL; DEL VILLAR; GARCÍA-GONZÁLEZ; MORENO; MORENO, 2015; LÓPEZ et al., 2016).

A pesar de todo, la importancia de la implementación de este modelo pedagógico no parece corresponderse con una inclusión real en la EF escolar de la mayoría de países en los que se ha difundido (ALMOND, 2010; STOLZ; PILL, 2016). Por ello, la utilización del modelo TGfU en la práctica docente, y más concretamente en la etapa de Educación Primaria, es todavía muy limitado, pudiéndose observar que en los contenidos relativos a los deportes de colaboración-oposición, la enseñanza de la técnica sigue siendo la prioridad en el tiempo de clase, no siendo frecuente el uso de estilos de enseñanza que promuevan la significatividad y la reflexión sobre estos aprendizajes deportivos (ROBLES; GIMÉNEZ; ABAD, 2011).

Por todo ello, esta investigación tiene como objetivo analizar el efecto de un programa de enseñanza comprensiva, basado en el cuestionamiento, para la mejora de la toma de decisiones del pase y del lanzamiento, en una unidad didáctica (UD) de baloncesto en Educación Primaria.

\section{MÉTODO}

\subsection{PARTICIPANTES}

La muestra del presente estudio estuvo compuesta por 37 alumnos de dos grupos diferentes de 6ํㅜ curso de un colegio de Educación Primaria, con edades comprendidas entre los 11 y 12 años ( $M=11.22 ; D T=.422)$. El grupo experimental estuvo formado por 20 alumnos y el grupo control por 17 alumnos. La intervención fue llevada a cabo por un mismo docente.

Inicialmente, fueron considerados un total de 50 alumnos, pero una vez aplicado el programa de intervención, no fueron tenidos en cuenta aquellos participantes que faltaron a 3 o más sesiones; los que faltaron a clase en la evaluación inicial o en la evaluación final; y aquellos que no tenían ninguna acción evaluada en algunas de las categorías de codificación del programa de intervención (pase y lanzamiento).

Fueron seguidas las directrices de la Declaración de Helsinki (2008), con respecto al consentimiento, confidencialidad y anonimato de los resultados.

\subsection{VARIABLES}

\subsubsection{Variable independiente}

La variable independiente de la presente investigación se corresponde con el programa de enseñanza comprensiva, basado en el cuestionamiento, para la mejora de la toma de decisiones en una UD de baloncesto. El programa estuvo formado por dieciocho sesiones 
de $E F$, de las cuales dos sesiones fueron de evaluación (una de evaluación inicial y otra de evaluación final), y dieciséis de desarrollo o aprendizaje.

El programa de intervención se llevó a cabo en el segundo trimestre, de acuerdo con la temporalidad prevista para este contenido en la programación didáctica del área de EF del centro. Los contenidos principales fueron el pase y el lanzamiento, pero también se trataron otros contenidos como el bote, los desplazamientos, los espacios, la defensa y el ataque. Las sesiones se organizaron atendiéndose al contenido principal a trabajar, de manera que la UD quedó secuenciada de la siguiente manera: en la primera y segunda se trabajó la posesión del balón en ataque y el bote; en la tercera y cuarta, el pase; en la quinta y sexta, el lanzamiento; en la séptima y octava, los espacios; en la novena y décima se trabajó la defensa; en las sesiones once y doce, el ataque; en las sesiones trece y catorce se trabajó de manera conjunta el ataque y la defensa; y en las sesiones quince y dieciséis se trabajaron de forma integrada, junto con el lanzamiento y el pase, el bote, los desplazamientos, el ataque y la defensa. En todas las sesiones se trabajó el pase y el lanzamiento, como contenido principal o como contenido secundario.

Para el diseño de las actividades del programa de intervención, fueron manipuladas las variables tácticas que permitían modificar el juego e ir progresando en la complejidad de éste (principios de juego, número de alumnos por equipo, nivel de oposición, tamaño del campo, canastas, balones y duración del juego). De este modo, inicialmente el número de componentes de un equipo era mínimo, existía superioridad numérica en ataque, el tamaño del terreno de juego no estaba limitado y no existía limitación de tiempo para conseguir el objetivo. Así, se favorecía la máxima participación de los alumnos, una mayor continuidad en el juego, una menor exigencia táctica y una mayor facilidad para la ejecución de la habilidad.

Cada sesión programada comenzó con un juego modificado de cinco minutos, que animaba a los alumnos a reflexionar sobre un problema específico, definido previamente por el profesor. A este juego, le continuaron tres juegos modificados de diez minutos cada uno, con mayor componente táctico, centrados en los principios básicos de juego de ataque y defensa (BAYER, 1992). En cada juego predominaron los juegos modificados por representación (e.g., mantener el control de la pelota mediante pases y recepciones mientras se progresa hacia la canasta, delimitando un espacio común para todos los grupos (reduciéndose el espacio progresivamente), consiguiendo punto el equipo que consiga diez pases seguidos sin perder el control de la pelota), complementándose a su vez por la práctica de juegos modificados por exageración (e.g., 4vs4 sin poder realizar pases hacia atrás). De esta manera permitimos a los alumnos vivenciar un número más elevado de situaciones específicas de juego en ese contexto real (SERRA-OLIVARES; GONZÁLEZ-VÍLLORA; GARCÍA-LOPEZ, 2011).

Dentro de este contexto de juegos modificados, la aplicación del cuestionamiento estuvo destinado a implicar cognitivamente al alumno. De esta manera, para cada juego modificado fue vinculado un cuestionamiento por parte del profesor, centrado en los principios técnico-tácticos presentes en la acción de juego a trabajar. Así, para asegurar la eficacia del cuestionamiento, se tuvieron en cuenta las cinco áreas que aseguran la calidad de la intervención del docente (estrategia, táctica, técnica, normas, aspectos psicológicos) (CLEMENTE, 2014). Igualmente, se formó al docente que implementó la unidad didáctica en diseñar las preguntas para el cuestionamiento atendiendo a: ¿qué?; ¿dónde?; ¿cuándo?; ¿por qué?; ¿quién?;y ¿cómo?) (GRIFFIN; BUTLER, 2005). El docente fue orientando el cuestionamiento atendiendo a las 
necesidades que acontecían durante el desarrollo de las actividades, relacionándolo con los objetivos y contenidos previstos para cada sesión (ver Tabla 1).

Tabla 1 - Sesiones, objetivos didácticos y orientaciones del cuestionamiento del programa de intervención

\begin{tabular}{|c|c|c|}
\hline Sesiones & Objetivos didácticos & Orientación del cuestionamiento \\
\hline $1-2$ & $\begin{array}{l}\text { Mantener la posesión del balón en ataque. } \\
\text { Concienciar de la importancia táctica del } \\
\text { bote de balón }\end{array}$ & $\begin{array}{l}\text { Tipos de bote en situaciones concretas; control } \\
\text { y protección del balón; adaptación del bote con } \\
\text { respecto a la posición del defensor }\end{array}$ \\
\hline $3-4$ & $\begin{array}{l}\text { Progresar hacia la meta contraria. } \\
\text { Concienciar de la importancia del pase. }\end{array}$ & $\begin{array}{l}\text { A qué hay que prestar atención antes de pasar; } \\
\text { tipos de pases en función de la posición del } \\
\text { compañero/s y defensor/es; colocación del defensor } \\
\text { en línea de pase; compañero libre de marca. }\end{array}$ \\
\hline $5-6$ & $\begin{array}{l}\text { Finalizar la jugada. } \\
\text { Realizar lanzamientos a canasta en las } \\
\text { mejores condiciones posibles. }\end{array}$ & $\begin{array}{l}\text { Momento del lanzamiento; posición del defensor con } \\
\text { respecto al lanzador; análisis de otras posibilidades } \\
\text { antes de lanzar. }\end{array}$ \\
\hline $7-8$ & $\begin{array}{l}\text { Ocupar espacios libres } \\
\text { Progresar hacia la meta contraria }\end{array}$ & $\begin{array}{l}\text { Lugar al que desplazarse sin balón; ubicación del } \\
\text { defensor/es; ubicación del compañero/s. }\end{array}$ \\
\hline $9-10$ & $\begin{array}{l}\text { Comprender elementos básicos de la } \\
\text { defensa (marcaje), para adaptar las } \\
\text { acciones ofensivas }\end{array}$ & $\begin{array}{l}\text { Dificultades para avanzar en ataque; ubicación de } \\
\text { los defensores; organización del ataque adaptado a } \\
\text { la posición de los defensores. }\end{array}$ \\
\hline $11-12$ & $\begin{array}{l}\text { Profundizar en elementos básicos de } \\
\text { ataque y contraataque }\end{array}$ & $\begin{array}{l}\text { Posición en situaciones sin balón; movimientos tras } \\
\text { robo; opciones de finalización de jugada;. }\end{array}$ \\
\hline $13-16$ & $\begin{array}{l}\text { Trabajar de manera integrada situaciones } \\
\text { de ataque y de defensa } \\
\text { Afianzar patrones aprendidos en sesiones } \\
\text { anteriores }\end{array}$ & $\begin{array}{l}\text { Opciones más eficaces en el juego de ataque; } \\
\text { análisis de situaciones diversas aprendidas (lanzar } \\
\text { o pasar; ocupar un lugar u otro en función de la } \\
\text { organización defensiva...) }\end{array}$ \\
\hline
\end{tabular}

Fuente: datos de los autores.

Atendiendo a estas orientaciones, por ejemplo, en una situación de 3vs1 en el que el objetivo era avanzar hacia la canasta contraria con el nivel más bajo de oposición, el cuestionamiento se orientó de la siguiente manera: ¿cómo debéis organizaros para poder avanzar hacia la canasta contraria? De esta manera, el docente pretendía guiar al alumno en tres niveles de cuestionamiento y análisis: tiempo, espacio y nivel de riesgo (MITCHELL; OSLIN; GRIFFIN, 2006).

El cuestionamiento se desarrolló de manera colectiva, con todo el alumnado, favoreciendo la reflexión en dos momentos diferentes: durante el desarrollo del juego y al finalizar la actividad. El tiempo de discusión con el alumnado no excedió de los 2 minutos, siguiendo las recomendaciones de Vickers (2007) para la aplicación del cuestionamiento. De la misma manera, el docente planteaba cuestionamientos individuales, en los casos en los que así se precisase. Este cuestionamiento fue planteado tras la acción del alumnado en las tareas desarrolladas.

Por otro lado, el grupo control llevó a cabo sus sesiones con la misma estructura y juegos que los del grupo experimental, pero sin la aplicación del cuestionamiento como herramienta de intervención del docente, limitándose a utilizar feedbacks prescriptivos para la corrección de las tareas.

\subsubsection{Variable dependiente e instrumento de medida}

La variable dependiente considerada en este estudio fue la toma de decisiones en las acciones de pase y lanzamiento. El instrumento de recogida de datos utilizado fue la 
observación sistemática indirecta y externa de la toma de decisiones, metodología que ha sido empleada en estudios anteriores para analizar la competencia táctica en situación de juego modificado (GUTIÉRREZ-DÍAZ; GONZÁLEZ-VÍLLORA; GARCÍA-LÓPEZ; MITCHELL, 2011). La observación de la toma de decisiones se realizó mediante el instrumento propuesto por Méndez-Giménez (2005), registrando el número de acciones apropiadas e inapropiadas del pase y del lanzamiento. Se registraron todas las acciones de pase y lanzamiento de cada uno de los alumnos que formaban ambos grupos. Para la valoración de la toma de decisiones se utilizó el componente toma de decisiones del citado instrumento, asignando un valor $1 \mathrm{a}$ decisiones apropiadas (e.g. para la acción de pase: el alumno trata de pasar a un compañero libre) y con un 0 a decisiones inapropiadas (e.g. para la acción de lanzamiento: lanzar cuando es más oportuno un pase a un compañero libre y adelantado).

Para el desarrollo de esta investigación fueron utilizados los siguientes medios audiovisuales y tecnológicos: un ordenador portátil acer ASPIRE 5742, una vídeo-cámara Sony HDRXR155, un trípode Hama Gamma Series.

\subsection{FIABILIDAD DE LA OBSERVACIÓN}

Para garantizar la fiabilidad de la observación, un profesor-observador conocedor del baloncesto, realizó un proceso de entrenamiento, en el que se utilizó una muestra superior al $10 \%$ de la muestra total. En la observación de todas las variables se alcanzaron unos valores de Kappa de Cohen intra-observador superiores a .81, valor a partir del cual se puede considerar como una concordancia casi perfecta (FLEISS; LEVI; CHO PAIK, 2003). Para garantizar la fiabilidad temporal de la medida, se desarrolló la misma codificación en dos momentos diferentes, con una diferencia temporal de diez días, obteniendo unos valores de Kappa de Cohen superiores a .85 .

\subsection{PROCEDIMIENTO}

Con el objeto de garantizar la aplicación correcta del modelo de enseñanza comprensiva, se aleccionó al docente que iba a desarrollar el programa, por parte de un experto. Al igual que en otros estudios (HARVEY et al., 2010; PRÁXEDES et al., 2016), el programa de formación se desarrolló durante tres sesiones, de una duración de una hora y media cada una. En la primera de ellas se abordaron los principios básicos del modelo TGfU; en la segunda se incidió sobre la aplicación de los juegos modificados; y en la última sobre la utilización del cuestionamiento en la formación de los alumnos. Estas sesiones de formación fueron desarrolladas por el investigador principal, con dilatada experiencia y amplio conocimiento del objeto de estudio. Igualmente, durante las sesiones iniciales de desarrollo de la UD, este investigador estuvo presente, en un lugar alejado y sin intervenir en la práctica, con el fin de valorar si los conocimientos adquiridos por el docente durante la formación fueron adecuados y si el programa se implementaba de manera correcta.

En un primer momento tuvo lugar la evaluación inicial, que se desarrolló en dos días diferentes para cada grupo de $6^{\circ}$ de primaria (experimental y control). En cada sesión de evaluación se jugaron cuatro partidos de 3vs3 en una pista exterior de baloncesto de 15x14 metros, evitándose la posibilidad de incidencia de agentes externos que pudieran interrumpir la 
práctica. De esta manera, todos los alumnos jugaron un partido de siete minutos de duración cada parte, con un minuto de descanso, con un total de catorce minutos para la evaluación de cada alumno. Para la grabación de los partidos, el cámara fue situada en una esquina de la pista, a tres metros de altura, garantizando un óptimo plano de visión de todo el terreno de juego. Entre la evaluación inicial y la final, se desarrollaron las dieciséis sesiones de aprendizaje de las que estaba compuesto el programa de intervención. Todos los alumnos desarrollaron las mismas actividades de aprendizaje. Los agrupamientos durante las sesiones fueron organizados por el profesor, en función al desarrollo y evolución de las actividades.

Seguidamente, tuvo lugar la evaluación final, que se llevó a cabo siguiendo el mismo procedimiento que la evaluación inicial, enfrentándose así los mismos equipos.

\subsection{ANÁLISIS ESTADÍSTICO}

Se utilizó el programa estadístico SPSS 24.0 para el análisis y tratamiento de los datos. Se examinó la normalidad de los datos a través de la prueba de Shapiro-Wilk y la homogeneidad de las varianzas a través de la prueba de Levene, como paso previo a la aplicación del programa de intervención. La evaluación de ambos estadísticos posibilitó el uso de estadística paramétrica y determinó que los grupos eran equivalentes.

Por ello, se realizó un análisis inferencial a través de un MANOVA de dos factores con medidas repetidas en un factor, con el fin de determinar el efecto sobre la interacción entre los 2 momentos de medición (pre-test y post-test) y entre los dos grupos (experimental y control). Para determinar cuál era el tamaño del efecto se utilizó el estadístico Eta al cuadrado parcial $\left(\eta_{\mathrm{p}}^{2}\right)$.

\section{RESULTADOS}

Los estadísticos multivariados sobre la interacción entre los 2 momentos de medición (pre-test y post-test) y el grupo (control y experimental) muestran estos efectos de interacción significativos, siendo el tamaño del efecto de una magnitud media (Lambda de Wilks=.596; $F=9.504, p=.001, \eta_{p}^{2}=.404$ ).

Como se puede comprobar en la Tabla 2, las comparaciones por pares en cada una de las fases del estudio determinan, en la medida de post-test, la existencia de diferencias significativas entre ambos grupos en la toma de decisiones, tanto en la acción de pase como en la de lanzamiento.

Tabla 2 - Comparación por pares de la toma de decisiones en el pase y el lanzamiento en ambos grupos para las mediciones de pre-test y post-test.

\begin{tabular}{|c|c|c|c|c|c|c|c|c|c|c|c|}
\hline \multirow[t]{2}{*}{ Acción } & \multirow[t]{2}{*}{$\mathrm{T}^{\prime}$} & \multicolumn{2}{|c|}{$\begin{array}{c}\text { (I) } \\
\text { Experimental } \\
\end{array}$} & \multicolumn{2}{|c|}{$\begin{array}{c}(\mathrm{J}) \\
\text { Control }\end{array}$} & \multirow{2}{*}{$\begin{array}{c}\text { Dif. de } \\
\text { medias (I-J) }\end{array}$} & \multirow{2}{*}{$\begin{array}{l}\text { Error } \\
\text { típ. }\end{array}$} & \multirow[t]{2}{*}{$p$} & \multirow{2}{*}{$\eta_{p}^{2}$} & \multicolumn{2}{|c|}{$\begin{array}{c}\text { IC 95\% } \\
\text { diferencias }\end{array}$} \\
\hline & & $\mathrm{M}$ & DT & $\mathrm{M}$ & DT & & & & & L.I. & L.S. \\
\hline \multirow{2}{*}{ Pase } & Pre & .582 & .213 & .688 & .164 & -.106 & .071 & .145 & .008 & -.250 & .039 \\
\hline & Post & .900 & .074 & .823 & .108 & .077 & .033 & .026 & .248 & .010 & .144 \\
\hline \multirow{2}{*}{ Lanzamiento } & Pre & .506 & .252 & .564 & .250 & -.059 & .092 & .526 & .001 & -.246 & .128 \\
\hline & Post & .958 & .079 & .801 & .182 & .157 & .048 & .003 & .144 & .059 & .255 \\
\hline
\end{tabular}




\section{DISCUSIÓN}

El objetivo del presente estudio fue analizar el efecto de un programa de enseñanza comprensiva, basado en el cuestionamiento, sobre la toma de decisiones del pase y del lanzamiento en una UD de baloncesto, en un contexto de Educación Primaria.

Tras la aplicación del programa de intervención, los resultados mostraron diferencias significativas entre los grupos en la toma de decisiones, en las acciones de pase y del lanzamiento, a favor del grupo experimental. Por tanto, se puede determinar que el programa de enseñanza, basado en el cuestionamiento, mejoró la comprensión del juego de los alumnos sometidos a la intervención.

En este sentido, cabe destacar que estos datos confirman la tendencia contrastada en investigaciones anteriores, en las que se comprobó que la aplicación de los principios metodológicos del modelo TGfU mejoraban la calidad de la toma de decisiones y la eficacia en las acciones técnico-tácticas en diferentes deportes (balonmano, GARCÍA-HERRERO; RUIZ PÉREZ, 2003; fútbol, GONZÁLEZ-VÍLLORA; GARCÍA-LÓPEZ; CONTRERAS; GUTIÉRREZ, 2010; GONZÁLEZ-VÍLLORA; GARCÍA-LÓPEZ; PASTOR; CONTRERAS, 2011; PRÁXEDES et al., 2017; baloncesto, ALLISON; THORPE, 1997; BALAKRISHNAN et al., 2011; CONTE; MORENO-MURCIA; PÉREZ; IGLESIAS, 2013).

García-González, Moreno, Gil, Moreno, y del Villar, (2014) y García-González et al. (2013) señalan la importancia que tiene el cuestionamiento como herramienta pedagógica, ya que, al igual que en el presente estudio, se manifiesta que la intervención realizada dentro del juego real y en la que se incluye la aplicación del cuestionamiento, tiene efectos positivos en la toma de decisiones de los alumnos.

En esta línea, en un estudio en voleibol, Broek, Boen, Claessens, Feys y Ceux (2011), compararon tres metodologías de enseñanza (tradicionales, comprensiva sin la aplicación de las preguntas y comprensiva con la aplicación de preguntas), cuyos resultados mostraron, en las variables decisionales del último grupo, valores más elevados que en los otros dos grupos. De la misma manera, en un estudio con futbolistas de categoría alevín (1112 años), Práxedes et al. (2016) concluyeron que los jugadores que recibieron una formación deportiva basada en el cuestionamiento mejoraron significativamente su capacidad para tomar decisiones.

Estos estudios demuestran que un cuestionamiento eficaz debe promover el pensamiento reflexivo de los estudiantes en la toma de decisiones. En consecuencia, a través del protocolo de aplicación del modelo de cuestionamiento dentro de los modelos comprensivos (TGfU), se puede comprobar la relación entre la centralización de la educación en el estudiante y su capacidad para llegar a la solución de forma independiente (PEARSON; WEBB, 2008). Así, el docente debe tratar de cumplir con la complejidad de las cuestiones, tratando de adaptarse al nivel de los alumnos, adaptando las preguntas al nivel de su lógica evolución progresiva en la comprensión del juego (FORREST; PEARSON; WEBB, 2006).

En el contexto de la Educación Primaria, han sido escasos los estudios que han analizado la aplicación del modelo TGfU para la mejora de los procesos cognitivos y decisionales de los alumnos. El cuestionamiento a desarrollar debe plantearse de manera estructurada, acorde a diferentes criterios recomendados por varios autores, tales como el tipo de cuestionamiento 
(GRIFFIN; BUTLER, 2005), las áreas de intervención (CLEMENTE, 2014) y los niveles del mismo (MITCHELL et al., 2006). Dado que el diseño del cuestionamiento en el presente estudio ha considerado estos criterios, los resultados obtenidos permiten interpretar que éste ha sido el causante de las mejoras obtenidas en el grupo experimental.

Por ello, se puede considerar el presente estudio como un estudio de interés para el profesorado de esta etapa educativa, al demostrarse que el cuestionamiento puede considerarse una herramienta pedagógica de gran utilidad para el desarrollo de las habilidades deportivas previstas en el currículo. No obstante, una mayor cantidad de estudios que profundicen en esta etapa educativa, son fundamentales para establecer afirmaciones más concluyentes.

\section{CONCLUSIONES Y APLICACIONES PRÁCTICAS}

Los resultados obtenidos en la presente investigación, ponen de manifiesto un efecto positivo del cuestionamiento para la mejora de la toma de decisiones de los alumnos participantes. Si se entiende que la toma de decisiones es uno de los pilares básicos del desarrollo de un juego, se recomienda que, desde la formación inicial, como es la etapa de Educación Primaria, se implementen modelos pedagógicos basados en el cuestionamiento para el desarrollo de las habilidades técnico-tácticas. El desarrollo del pensamiento táctico debe orientarse hacia un aprendizaje comprensivo que permita a los alumnos mejorar sus conocimientos de manera explícita. Para ello será necesario diseñar tareas que propicien en los alumnos la posibilidad de tomar sus propias decisiones.

Por tanto, se considera que con la inclusión del modelo TGfU en las programaciones educativas, modificando los condicionantes de la práctica y proponiendo un cuestionamiento eficaz, los alumnos no solo son capaces de desarrollar un entendimiento de los aspectos técnicos y tácticos de un juego, sino que mejorarán además su capacidad para tomar decisiones y por lo tanto su aprendizaje. En este sentido, si bien es cierto que la aplicación de este modelo también nos sirve para diagnosticar el juego táctico, no podemos olvidar que, dentro de las áreas educativas, los docentes de EF se encuentran con numerosos obstáculos a la hora de impartir lo distintos contenidos.

Por lo tanto, existe la necesidad de utilizar modelos alternativos que favorezcan un desarrollo adecuado del proceso de enseñanza-aprendizaje, ya que tradicionalmente se ha obviado todo lo referente a los procesos decisionales, creando un cierto rechazo a los enfoques tácticos por parte de alumnos y docentes.

\section{REFERENCIAS}

ALLISON, Stuart; THORPE, Rod. A comparison of the effectiveness of two approaches to teaching games within physical education. A skills approach versus a games for understanding approach. The British Journal of Physical Education, v.28, n. 3, p. 9-13, 1997.

ALMOND, Len. Forward: Revisiting the TGfU brand. In: BUTLER, J.; GRIFFIN, L. (ed.) More teaching 
BALAKRISHNAN, Malathi; RENGASAMY, Shabeshan; AMAN, Mohd S. Teaching game for understanding in physical education: A theoretical framework and implication. ATIKAN, v. 1, n. 2, p. 201-214, 2011.

BAYER, Claude. La enseñanza de los juegos deportivos colectivos. Barcelona: Hispano Europea, 1992.

BROEK, Gert V; BOEN, Filip; CLAESSENS, Manu; FEYS, Jos; CEUX, Tanja. Comparison of three instructional approaches to enhance tactical knowledge in volleyball among university students. Journal of Teaching and Physical Education, v. 30, n. 4, p. 375-392, oct. 2011.

BUNKER, David; THORPE, Rod. A model for the teaching of games in secondary schools. Bulletin of Physical Education, v. 18, n. 1, p. 5-8, 1982.

BUTLER, Joy; OSLIN, Judith L; MITCHELL, Sthephen A; GRIFFIN, Linda. The way forward for TGfU: Filling the chasm between theory and practice. Physical \& Health Education Journal, v. 74, n. 1, p. 6-12, 2008.

CLEMENTE, Felipe M. Uma visão integrada do modelo teaching game for understanding: Adequando os estilos de ensino e questionamento à realidade da educação física. Revista Brasileira de Ciências do Esporte, v. 36, n. 2, p. 587-601, abr/jun 2014.

CONTE, Luis; MORENO-MURCIA, Juan A; PÉREZ, Gabino; IGLESIAS, Damián. Comparación metodología tradicional y comprensiva en la práctica del baloncesto. Revista Internacional de Medicina y Ciencias de la Actividad Física y el Deporte, v. 13, n. 51, p. 507-523, sep. 2013.

FLEISS, Joseph L.; LEVI, Bruce; CHO PAIK, Myunghee. Statistical methods for rates and proportions. New York: Wiley, 2003.

FORREST, Gregory J; PEARSON, Philip J; WEBB, Paul I. Teaching Games for Understanding (TGfU); a model for pre service teachers. In: INTERNATIONAL COUNCIL FOR HEALTH, PHYSICAL EDUCATION, RECREATION, SPORT AND DANCE (ICHPER), 1. [PROCEEDINGS], Wellington: Oceanic Congress, oct. 2006. p. 1-10.

GARCÍA-GONZÁLEZ, Luis; MORENO, Alberto; GIL, Alexander; MORENO; María P; DEL VILLAR, Fernando. Effects of decision training on decision making and performance in young tennis players: an applied research. Journal of Applied Sport Psychology, v. 26, n. 4, p. 426-440, abr. 2014.

GARCÍA-GONZÁLEZ, Luis; MORENO, María P; MORENO, Alberto; GIL, Alexander; DEL VILLAR, Fernando. Effectiveness of a video-feedback and questioning programme to develop cognitive expertise in sport. PLoS One, v. 8, n. 12, p. e82270, dec. 2013.

GARCÍA-HERRERO, Juan A; RUÍZ-PÉREZ, Luis M. Análisis comparativo de dos modelos de intervención en el aprendizaje del balonmano. Revista de psicología del deporte, v. 12, n. 1, p. 55-66, 2003.

GIL, Alexander; DEL VILLAR, Fernando. Aplicación de un programa de entrenamiento decisional, en tiempo real de juego, para la mejora de rendimiento táctico individual del deportista. In: GARCÍA-GONZÁLEZ, Luis; DEL VILLAR, Fernando. Entrenamiento táctico y decisional en el deporte. Madrid: Síntesis, 2014. p. 132-146. 
GIL, Alexander; DEL VILLAR, Fernando; GARCÍA-GONZÁLEZ, Luis, MORENO, Alberto; MORENO, María P. Effectiveness of video feedback and interactive questioning in improving tactical knowledge in volleyball. Perceptual and Motor Skills, v. 121, n. 3, p. 635-653, nov. 2015.

GONZÁLEZ-VÍLLORA, Sixto; GARCÍA-LÓPEZ, Luís M; CONTRERAS, Onofre R; GUTIÉRREZ, David. Estudio descriptivo sobre el desarrollo táctico y la toma de decisiones en jóvenes jugadores de fútbol (12 años). Revista Infancia y Aprendizaje, v. 33, n. 4, p.489-501, jan. 2010.

GONZÁLEZ-VÍLLORA, Sixto; GARCÍA-LÓPEZ, Luís M; PASTOR, Juan C; CONTRERAS, Onofre. Conocimiento táctico y toma de decisiones en jóvenes jugadores de fútbol (10 años). Revista de Psicología Del Deporte, v. 20, n. 1, p. 79-97, 2011.

GRAY, Shirley; SPROULE, John. Developing pupils' performance in team invasion games. Physical Education and Sport Pedagogy, v. 16, n. 1, p. 15-32, jan. 2011.

GRIFFIN, Linda L. Improving net/wall game performance. Journal of Physical Education, Recreation and Dance, v. 67, n. 2, p. 34-37, feb. 1996.

GRIFFIN, Linda; BUTLER, Joy. Teaching games for understanding: theory, research, and practice. Champaign, IL: Human Kinetics, 2005.

GRIFFIN, Linda L; BROOKER, Ross; PATTON, Kevin. Working towards legitimacy: two decades of teaching games for understanding. Physical Education and Sport Pedagogy, v. 10, n. 3, p. 213-223, nov. 2005.

GRIFFIN, Linda L; MITCHELL, Stephen A; OSLIN, Judith L. Teaching sports concepts and skills: a tactical games approach. Leeds: Human Kinetics, 1997.

GUTIÉRREZ, David; GARCÍA-LÓPEZ, Luis M. Assessment of primary school students' decisionmaking related to tactical contexts. Journal of New Approaches in Educational Research, v. 1, n. 1, p. 7-12, jul. 2012.

GUTIÉRREZ-DİAZ, David; GONZÁLEZ-VÍLLORA, Sixto; GARCÍA-LÓPEZ, Luis M; MITCHELL, Stephen. Differences in decision-making development between expert and novice invasion game players. Perceptual and Motor Skills, v. 112, n. 3, p. 871-888, jan. 2011.

HA, Amy S; WANG, Lijuan; COLLINS John. Perceptions of Hong Kong physical education teachers on Teaching Games for Understanding: Implications for continuing professional development. Educational Research Journal, v. 29, n. 1-2, p. 91-110, 2014.

HARVEY, Stephen; CUSHION, Christopher J.; MASSA-GONZÁLEZ, Ada N. Learning a new method: Teaching Games for Understanding in the coaches' eyes. Physical Education and Sport Pedagogy, v. 15, n. 4, p. 361-382, oct. 2010.

HARVEY, Stephen; GITTINS, Christopher. Effects of integrating video-based feedback into a Teaching Games for Understanding soccer unit. Ágora para la Educación Física y el Deporte, v. 16, n. 3, p. 271-290, sep. 2014.

HOPPER, Tim. Teaching games for understanding: The importance of students emphasis over contents emphasis. Journal of Physical Education, Recreation \& Dance, v. 73, n. 7, p. 44-48, sep. 2002.

KIRK, David; BROOKER, Ross; BRAIUKA, Sandy. Teaching games for understanding: A situated perspective on student learning. In: AMERICAN EDUCATIONAL RESEARCH ASSOCIATION ANNUAL MEETING. [PROCEEDINGS]. New Orleans, p. 24-28, apr. 2000. 
LAUNDER, Alan G. Play practice: The games approach to teaching and coaching sports. Champaign, IL: Human Kinetics, 2001.

LIGHT, Richard; FAWNS, Rod. Knowing the game: Integrating speech and action in games teaching through TGfU. Quest, v. 55 n. 2, p. 161-176, 2003.

LÓPEZ, Ismael; PRÁXEDES, Alba; DEL VILLAR, Fernando. Effect an intervention teaching program, based on TGfU model, on the cognitive and the execution variables, in the physical education context. European Journal of Human Movement, v. 37, p. 88-108, 2016.

MAHLO, Friedrich. 0 acto táctico no jogo. Lisboa: Compendium, 1970.

MCNEILL, Michael C; FRY, Joan M; WRIGHT, Stephen C; TAN, Clara WK; TAN, Stephen KS; SCHEMPP, Paul G. "In the local context": Singaporean challenges to games teaching on practicum. Sport, Education and Society, v. 9, p. 3- 32, 2004.

MÉNDEZ-GIMÉNEZ, Antonio. Hacia una evaluación de los aprendizajes consecuentes con los modelos alternativos de iniciación deportiva. Tándem, Didáctica de la educación física, v. 17, p. 38-58, 2005.

MITCHELL, Stephen A; OSLIN, Judith L; GRIFFIN, Linda L. Teaching sport concepts and skills: A tactical games approach. Champaign, IL: Human Kinetics, 2006.

PEARSON, Phil; WEBB, Paul. Developing effective questioning in Teaching Games for Understanding (TGFU). In: ASIA PACIFIC SPORT IN EDUCATION CONFERENCE, 1. [PROCEEDINGS...]. Adelaide, Jan. 2008, p. 1-9.

POULTON, E. On prediction in skilled movement. Psicological bulletin, v. 54, p. 467-478, 1957.

PRÁXEDES, Alba; GARCÍA-GONZÁLEZ, Luis; MORENO, Álvaro; MORENO, María P; MORENO, Alberto. Application of an intervention program to improve tactical understanding in indoor football: a study conducted in an educational context. Movimento, v. 22, n. 1, p. 51-62, jan./mar. 2016.

PRÁXEDES, Alba; MORENO, Alberto; SEVIL, Javier; GARCÍA-GONZÁLEZ, Luis; DEL VILLAR, Fernando. The effects of a comprehensive teaching program on dribbling and passing decisionmaking execution skills of young footballers. Kinesiology, v. 49, n. 1, p. 74-83, 2017.

ROBLES, José; GIMÉNEZ, Francisco J.; ABAD, Manuel T. Metodología utilizada en la enseñanza de los contenidos deportivos durante la E.S.O. Revista Internacional de Medicina y Ciencias de la Actividad Física y el Deporte, v. 10, n. 41, p. 35-57, 2011.

SALTER, David G. Teaching games and sport in the New Zealand Health and Physical Education Curriculum. Journal of Physical Education, v. 32, n. 2, p. 4-7, 1999.

SERRA-OLIVARES, Jaime; GONZÁLEZ-VÍLLORA, Sixto; GARCÍA-LÓPEZ, Luis M. Comparación del rendimiento de juego de jugadores de fútbol de 8-9 años en dos juegos modificados 3 contra 3. Cuadernos de Psicología del Deporte, v. 11, n. 2, p. 77-91, jun. 2011.

SINGLETON, Ellen. From command to constructivism: Canadian secondary school physical education curriculum and teaching games for understanding. Curriculum Inquiry, v. 39, n. 2, p. 321-342, mar. 2009.

STOLZ, Steven A; PILL, Shane. A narrative approach to exploring TGfU-GS. Sport, Education and Society, v. 21, n. 2, p. 239-261, 2016. 
TALLIR, Isabel B; LENOIR, Matthieu; VALCKE, Martin; MUSCH, Eliane. Do alternative instructional approaches result in different game performance learning outcomes? Authentic assessment in varying game conditions. International Journal of Sport Psychology, v. 38, n. 3, p. 263-282, 2007.

THORPE, Rod; BUNKER, David; ALMOND, Len. A change in focus for the teaching of games. In: PIERON, Maurice; GRAHAM, George. THE 1984 OLYMPIC SCIENTIFIC CONGRESS [PROCEEDINGS], 6. Sport Pedagogy. Champaign, IL: Human Kinetics, 1986. p. 163-169.

ÚBEDA-COLMENAR, Joan; MONFORTE, Javier; DAVID-DAVID, José. Percepción del alumnado sobre una Unidad Didáctica de enseñanza comprensiva de los juegos deportivos de invasión en Educación Física. Retos, v. 31, p. 275-281. 2017.

VICKERS, Joan N. Perception, cognition, and decisión training: The quiet eye in action. Champaign, IL: Human Kinetics, 2007.

VICKERS, Joan N; REEVES, M; CHAMBERS, Kristine L; MARTELL Steve. Decision training: Cognitive strategies for enhancing motor performance. In:

WILLIAMS, A Mark; HODGES, Nicola. Skill acquisition in sport: Research, theory and practice. New York: Routledge, 2004. p. 103-120.

WEBB, Paul; PEARSON, Phil; FORREST, Greg. Teaching Games for Understanding (TGfU) in primary and secondary physical education. In: INTERNATIONAL CONFERENCE FOR HEALTH, PHYSICAL EDUCATION RECREATION, SPORT AND DANCE, 1st. [PROCEEDINGS]. Oceanic Congress, Wellington, New Zealand, oct. 2006

WRIGHT, Steven; MCNEILL, Michael; BUTLER, Joy. The role that socialization can play in promoting teaching games for understanding. Journal of Physical Education, Recreation \& Dance, v. 75, n. 3, p. 46-52, 2004.

\section{Apoyo:}

Este estudio se ha realizado gracias a la aportación de la Consejería de Economía e 